\title{
Simon Magus in der altchristlichen Literatur.
}

\author{
Von Hans Waitz in Darmstadt.
}

Wie der Simon Magus in der altchristlichen Legende die Fähigkeit hat, sein Angesicht zu verwandeln, ${ }^{x}$ so erscheint er auch in:der altchristlichen Literatur in den verschiedenartigsten Gestalten. Bald tritt er als Magier und Pseudomessias auf, bald als eine Karrikatur des Apostels Paulus und der Antichrist; bald als Gnostiker und Archihäretiker, bald als Vertreter des Marcionitismus, ja sogar des Heidentums.

Demgemäß wird er auch in den wissenschaftlichen Bearbeitungen auf das verschiedenste dargestellt. Entweder wird er als geschichtliche Persönlichkeit überhaupt nicht anerkannt und als ein Phantasiegebilde bezeichnet, in dem als geschichtlicher Kern niemand anders als der Apostel Paulus stecke, oder er wird anerkannt, zugleich aber identifiziert mit einem cyprischen Juden Simon, von dem Josephus erzählt, ${ }^{2}$ oder unterschieden von dem Gnostiker Simon, dessen die Häreseologen gedenken. Und mannigfaltig wie die Darstellungen des Simon Magus sind auch die Versuche, die Widersprüche zu erklären, die sein Bild in der altchristlichen Literatur aufweist.

Wenn ich das geschichtliche Problem, das damit gegeben ist, von neuem aufnehme, so gehe ich von" denjenigen Schriften aus, die mit Recht als der Schlüssel dazu bezeichnet worden sind, von den sogen. Pseudoklementinen. Indem ich im allgemeinen dafür auf meine Abhandlung hierüber verweise, die in den. Texten und Untersuchungen N. F. X, 4. erschienen ist, bemerke ich nur dies, daß ich insofern eine neue Grundlage für die vorliegende Untersuchung gewonnen habe, als ich für die pseudoklementinischen Homilien und Rekognitionen bezw. deren gemeinsame Grundschrift 3 hauptsächlich zwei Quellenschriften

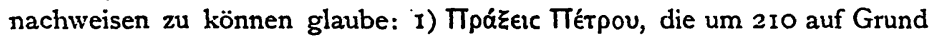

I Vgl. Rec. 2, 9 (10, 56) Hom. 2, 32.34 (20, 14).

2 Vgl. Jos. Ant. XX, 7, 2.

$3 \mathrm{Sie}$ ist nach meiner Annahme zwischen 220-230 in synkretistisch gerichteten, aber katholisch denkenden Kreisen der römischen Gemeinde entstanden.

Zeitschr. f. d. neutest. Wiss. Jahrg. V. Ig04. 
ältercr Petrusakten von einem katholischen Kleriker in Antiochien verfalt sind; 2) Knpúruata MEтpou, eine Schrift antipaulinischen Charakters, die bald nach 135 in Cäsarea in einer judenchristlich-gnostischen Sekte entstanden und später unter Benutzung von Justins Antimarcion in antimarcionitischem Interesse überarbeitet worden ist. Indem der Verfasser des Klemensromanes, der klementinische Redaktor, die Erzählung jener Petrusakten, deren Helden der Apostel Petrus und der Magier Simon

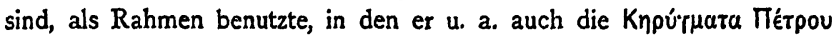
einfügte, übertrug er, was hier von Paulus bezw. Marcion gesagt war, auf den Magier Simon, der so nicht nur Paulus, sondern auch Marcion, ja zugleich auch Vertreter der heidnischen Volksreligion und Philosophie wurde. Hat daher das Simonbild der Klementinen zweifellos, wie die Tübinger Schule erkannt und nachgewiesen hat, an einzelnen Stellen paulinische Züge, so erklärt sich doch dies nicht auf dem Wege der Tendenzkritik, sondern allein auf dem der Literarkritik. Und es ist nicht nur unrichtig, den Simon in den Klementinen als eine Karrikatur des Paulus aufzufassen, sondern es läßt sich mit den Mitteln der Quellenkritik nachweisen, daß der Magier Simon hier niemals als ein Gegenstück zu dem großen Heidenapostel gedacht worden ist.

Dürfen wir daher von dem Simon, qui et Paulus absehen, den erst kürzlich noch Hilgenfeld für sich in Anspruch genommen hat, ${ }^{x}$ so müssen wir um so ernstlicher an die Aufgabe herantreten, die geschichtliche Gestalt dieses Simon Magus sowie die Wandlungen darzulegen, die sie im Laufe der ersten Jahrhunderte durchgemacht hat.

I.

Wenn die Tübinger Schule ihrer tendenziösen Auffassung gemäß ein Interesse daran hatte, die Existenz eines Magus Simon zu leugnen, ${ }^{2}$ so ist sie doch selber dazu zurückgekehrt, sie anzuerkennen. ${ }^{3}$ Und es muß auch mit ihm als einer geschichtlichen Größe gerechnet werden.

Das übereinstimmende Zeugnis von einem Magier Simon, der in Samarien wirkte, wie es in Act. 8 bezw. in deren Quelle,4 bei dem aus Samarien stammenden Justin, Apol. I 26. 56; Dial. c. Tryph. 120, bei Irenäus, adv haer. I 23; Tertullian, de anima 34; Hippolyt, Philos. VI 7

× Vgl. Zeitschr. f. w. Th. 1903 S. 330.

2 Vgl. Lipsius in dem Artikel Simon in Schenkels Bibellexikon V 301 ff., sowie Lüdemann, Prot. Kirchenzeit. 1887 S. 960 f.

3 Vgl. Hilgenfeld Ketzergeschichte des Urchristentums 1884. 163 ff. Lipsius Apokryphe Apostelgeschichten II I 36.56.

4 Vgl. dazu meine Abhandlung über die Pseudoklementinen in T. u. U. NF X. 4, S. 223 f. 
Euseb, h.e. II, 13 ff., Epiphanius adv. haer. 2 I; Theodoret haer. fab. I, I. Const. ap. VI 7 sowie in den Petrusakten ${ }^{x}$ vorliegt, kann nicht anders als eine historische Überlieferung bezeichnet werden. Dies um so sicherer, als es nicht auf eine gemeinsame Quelle zurückgeführt werden kann.

Dazu beweist die Existenz einer Sekte der Simonianer, daß auch ein Simon existiert hat, auf den jene Sekte zurückgeht. So erzälltt schon um die Mitte des 2. Jahrhunderts Justin in seiner I. Apologie den Römern, daß-fast alle Samaritaner, wenige aber auch unter den andern Völkern den Magier Simon, der zur Zeit des Klaudius (4I-54) nach Rom gekommen sei, als ersten Gott bekannt und verehrt hätten (Apol 1, 26). An einer anderen Stelle derselben Schrift wiederholt er diese Angabe, indem er hinzufügt, daß auch noch zu seiner Zeit viele zu den Anhängern Simons gehörten ( $I, 56 f$.). In seinem Dialog c. Tryph. I20 bezieht er sich ausdrücklich auf diese Ausführungen und bemerkt dazu, daß er sich bei seinen Angaben von keinen Rücksichten auf seine Landsleute, die Samaritaner, habe leiten lassen. Andernfalls - das ist der Sinn der Stelle - hätte er in ihrem Interesse besser verschwiegen, daß sie ihrem Landsmann, dem Magier Simon, anhingen. Sonach muß noch zur Zeit Justins die göttliche Verehrung Simons in Samarien volkstümlich gewesen sein. -

Noch nicht ein halbes Jahrhundert später gibt uns Irenäus weitere Kunde von der Existenz einer religiösen Gemeinschaft der Simonianer. Ist ex auch in seiner Darstellung des Lebens und der Lehre Simons (adv. haer. I, 23, I-3) von Justin, u. zw. von dessen verlorenem Syntagma wider alle Häresien, abhängig, so hat er doch seine Kenntnis von den Simonianern (I, 23, 4) anderswoher geschöpft. Denn wenn er hier von Götterbildern des Simon und der Helena redet, welche die Simonianer nach Zeus- und Atheriebilder verfertigten und verehrten, ${ }^{2}$ so hat er diese Bemerkung nicht dem Justin entnommen, der sie, falls er sie kannte, sicher nicht unterdrückt hätte, wo er von der göttlichen Verelhrung Simons durch eine Bildsäule mit der Inschrift Simoni deo sancto redet (Apol. 1, 26. .56).3 Also hat noch zur Zeit des Irenäus eine Sekte der Simonianer bestanden, die nach seiner Darstellung wesentlich okkultistischen Charakter hatte.

I Vgl. Rec. 2, 7 Hom. 2, 22, Act. Petr. c. Sim. ed. Lipsius et Bonnet I Lips. I89 I 48. 49 .

2 Davon spricht auch Hippolyt, Philos. VI, 20, doch offenbar nach Irenãus.

3 Diese Bildsäule war jedoch nicht dem Simon, sondern der altsabinischen Gottheit Semo Sancus geweiht. 
Dasselbe bezeugt Tertullian (de anima 57). Auch er kennzeichnet sie als Okkultisten, die sich mit Geisterbeschwörungen abgeben. Diese eigenartige Notiz bewcist übrigens, daß er seine Kenntnis nicht aus Justin oder Irenäus geschöpft hat, und bezeugt uns also von neuem durchaus selbständig die Existenz einer simonianischen Sekte.

Als dritter Abendländer kommt Hippolyt in Betracht (Philos. VI, 19). Hat er auch seine Kenntnis z. T. aus Irenäus, so weiß er doch, da@ es Anhänger des Simon und der Helena ếwc vôv gibt. Dazu bringt er aus einem Buch, das er dem Simon beilegt und das jedenfalls in seiner Sekte kanonische Bedeutung hatte, gröliere Auszigge." Sicher hat auch er noch Simonianer gekannt.

Alle drei Häreseologen des Abendlandes lassen es in der Schwebe, wo diese Sekte bestand. Doch liegt es nahe, an das Abendland, besonders Rom zu denken. Namentlich kann man sich bei Hippolyt nur Rom als den Boden denken, auf dem er sie und ihr beiliges Buch hatte kennen lernen. ${ }^{2}$

Aber auch im Morgenland hat sie noch um dieselbe Zeit $( \pm 200)$ bestanden.

Schon Celsus, der in der 2. Hälfte des 2. Jahrhunderts und wohl im Orient (Alexandrien?) lebte, hat sie gekannt (vgl. Orig. c. Cels. V, 62). Auch Clemens Alex. hat von ihr Kenntnis gehabt (Strom. II, II 52) und ebenso Origenes (c. Cels. $\mathrm{r}, 57$ ). Doch ist letzterer der Meinung, da es in der ganzen Welt kaum mehr als 30 gebe; ja an einer andern Stelle c. Cels. VI, II) spricht er die Vermutung aus, da@ überhaupt keine Anhänger Simons mehr existierten. Die Didaskalia $(\mathrm{VI}, 9)$ weiß jedoch noch von solchen. Auch zur Zeit des Epiphanius (adv. haer. 21) ebenso des Euseb (h. e. II I, 12) muß noch die Sekte bestanden haben; zur Zeit des Theodoret (haer. fab. I I) war sie aber bereits im Morgenlande erloschen. Im Abendlande ist die pseudocyprianische Schrift de rebaptismate 16 der letzte Zeuge für sie, indem sie erzählt, daß die Simonianer bei der Taufe Feuer über dem Wasser erscheinen ließen.

Nach alledem kann nicht geleugnet werden, dab es eine simonianische Sekte gab, welche nach Justin besonders in Samarien weit verbreitet und noch in der 2. Hälfte des 2. Jahrhunderts so zahlreich war, daß ein Heide wie Celsus Kenntnis von ihr hatte.

I Vgl. darüber unten S. $140 \mathrm{f}$.

2 Die apostol. Konstitutionen VI, 9 bemerken ausdrücklich das Aufkommen der Simonianer in Rom. Doch beruht diese Bemerkung, die in der Didaskalia fehlt, aur auf einem Schluß aus der vorhergebrachten Erzählung von dem Ende Simons in Rom. 
Thre Existenz hat die Existenz einer Persönlichkeit zur Voraussetzung, nach der sie sich nannte. Damit bestätigt sie uns, was uns schon die patristischen Zeugnisse bewiesen, daß es einen Simon gegeben hat, auf den sie sich zurückführte. Seine Geschichtlichkeit leugnen kann daher nicht anders denn mit Harnack ${ }^{x}$ als eine schwere Verirrung der Kritik bezeichnet werden. Sicher wäre sie überhaupt nicht in Frage gezogen worden, wenn man sich nicht durch die Auffassung des Simon in den Klementinen als des Paulus dazu hätte verleiten lassen.

Wer aber war der Simon, den jene Sekte als ihr Haupt verehrte?

Ihre ursprünglich zahlreiche Verbreitung in Samarien, die uns Justin bezeugt, sowie ihr okkultistischer Charakter lassen von vorn herein vermuten, $\mathrm{da} B$ er kein anderer war als jener samaritanische Magier Simon, der uns in der altchristlichen Literatur genugsam begegnet ist.

Das bestätigen uns aber die patristischen Zeugnisse selber.

Wie man auch über den Bericht Act 8 urteilen mag, so wird man ihn doch - wenn man ihm nicht mehr eine antipaulinische Tendenz zuerkennen kann - als. Urkunde wenigstens dafür betrachten dürfen, daßs zur Zeit der Apostel in Samarien ein Magier namens Simon lebte, der großes Aufsehen erregte.

Unabhängig hiervon bezeugt auch Justin (Apol. I 26. 56), daß er eine sichere Überlieferung von seinem Landsmann gehabt hat, der aus dem Flecken Gittä in Samarien stamme und unter dem Kaiser Klaudius nach Rom gekommen sei. ${ }^{2}$

Ein weiteres Zeugnis für diesen Simon, den Zeitgenossen der Apostel, das sowohl von der Apostelgeschichte als auch von Justin unabhängig ist, gibt Clemens Alex. an einer - allerdings viel umstrittenen - Stelle (Strom. VII, 17, 107) ${ }^{3}$ die, wörtlich genommen, zu dem Nonsens führt, dab Marcion ein Altersgenosse des Petrus war, und die daher nur ironisch in

I Doginengeschichte I1, 179. Anm. 1.

2 Vgl. Iren. adv. haer. I, 23, 1; Tert. Apol. 13; Eus. h. e. II 13; Epiphan. adv. haer. 21.

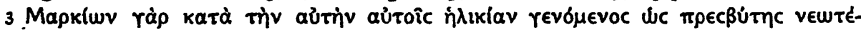

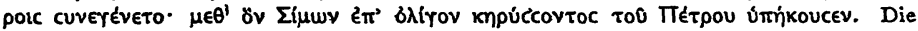
Korrektur von Is. Voß u. Pearson, $\mu \in \theta^{\prime} \bar{w} v$ oder von Baraterius $\mu \in \theta^{\prime}$ oû statt $\mu \in \theta^{\prime} \delta v$ ist willkürlich; die Auslegung des Valesius von $\mu \epsilon \tau \dot{\alpha}=$ ante ist unrichtig. Die Beziehung der Worte $\mu \in \theta^{2} \delta v$ auf Basilides - durch Annahme einer größeren Parenthese -, wie sie von Dodwell, diss. in Iren. 19 vorgeschlagen wird, ist gekünstelt und stört die ganze Symmetrie des Gedankengangs, wonach zu jedem der von Klemens hauptsächlich bekämpften Häretiker, Basilides, Valentin und Marcion, ein zweiter und älterer als Sektenhaupt hinzugefügt wird. Demnach kann hier Simon im Sinne des Klemens nur als Lehrer des Marcion betrachtet werden, ähnlich wie er bei Justin als der Urheber aller Ketzereien und so auch Marcions erscheint. 
dem Sinne genommen werden kann: Alle Häretiker suchen ihre Lehren bis in die apostolische Zeit hinauf zu datieren. So beruft sich Basilides auf Glaukias, den Dolmetsch des Petrus, Valentinus auf Theodas, den Bekannten des Paulus, so auch Marcion auf Simon, der die Predigt des Petrus hörte. Wenn Klemens den Simon als Zuhörer der Predigten des Petrus bezeichnet, muf er einen andern Bericht als Act 8 im Auge haben, da hier Simon nur als Zuhörer der Predigten des Philippus und als Gegner des Petrus charakterisiert ist. Diesen Bericht kann er aber auch nicht aus Justin geschöpft haben, welcher von einem Zusammentreffen des Simon und Petrus nichts weiß.

Sehen wir von allen weiteren Nachrichten bei den Kirchenvätern $a b$, unter denen noch besonders Epiphanius selbständige Kenntnis verrät, so werden wir nicht daran zweifeln, daß der hier genannte Simon der Stifter der simonianischen Sekte ist.

Demgegenüber hat Kreyenbühl ${ }^{2}$ für diese Sekte, wohl auch einen Gründer gefordert. Indem er aber ihn in dem Verfasser des simonianischen Buches àmópacic $\mu \in \gamma a ́ \lambda \eta$ sieht, aus dem Hippolyt Auszüge gibt, versetzt er ihn in den Beginn des 2. Jahrhunderts und unterscheidet ihn von dem Simon, den Justin als Urheber der simonianischen Häresie nennt. Nun aber ist es schon an und für sich sehr unwahrscheinlich, dab ein Buch wie die ámópacıc $\mu \in \gamma \alpha \dot{\lambda} \lambda \eta$, in dem Simon als Inkarnation der Gottheit erscheint, ihn selber zum Verfasser habe. Völlig unhaltbar aber erscheint die Vorstellung, daf dies Buch schon am Anfang des 2. Jahrhunderts vorhanden gewesen sei, wenn man das Schweigen der Kirchenväter, besonders des Justin und Irenäus, in Betracht zieht. Die Hypothese aber, diese beiden Häreseologen hätten ihre Nachrichten von der simonianischen Gnosis mit Hippolyt aus der ámópacıc geschöpft, erweist sich gerade da, wo man auf sie die Probe machen kann, als unhaltbar; denn nachweislich haben sie aus den uns durch Hippolyt bekannten Abschnitten der árópacıc nichts genommen. Dann aber haben sie dies Buch überhaupt nicht gekannt, dies um so sicherer, als Hippolyt in dem, was er mit Justin und Irenäus gemeinsam hat, nicht aus der ámópacıc, sondern aus Irenäus geschöpft hat. Wenn endlich Kreyenbühl a. a. O. 20r zur Begründnng seiner Hypothese einen Anachronismus darin zu erkennen vermeint, daß nach Justin Simon, der Lehrer des Menander dessen Schüler die Zeitgenossen des Ápologeten waren (Apol. I, 26), ein Zeitgenosse der Apostel gewesen sei, so können wir einen chronologischen

I Evangelium der Wahrheit. Berlin 1900, I99ff., 217ff. 
Widerspruch nicht darin finden und müssen es überhaupt ablehnen, aus der Bezeichnung des Schülerverhältnisses chronologische Daten zu gewinnen.

Aber auch angenommen, Justin, der in der I. Hälfte des 2. Jahrhunderts in Samarien lebte, habe das Verhältnis des - von Kreyenbühl angenommenen - in dieser Zeit lebenden Gnostikers Simon zu dem Zeitgenossen der Apostel, dem Magier Simon, nicht mehr so deutlich erkannt wie Kreyenbühl, so bleibt doch noch die Nachricht der Apostelgeschichte und des Clemens Alexandrinus übrig. Wir werden daher an der Identität des Magiers und des Oberhaupts der simonianischen Sekte festhalten, wenn wir auch nicht verkennen, daß der Magier und der Gnostiker Simon sehr verschiedene Züge an sich tragen (s. u. S. I3 f.).

Wie aber verhält sich dieser Magier Simon zu dem Magier Simon von dem Josephus Antiqu. XX, 7, 2 erzählt, daß er seinen Freund, den römischen Prokurator Felix, mit Drusilla, der Frau des Königs Azizus von Emesa, verkuppelt habe?

Nach Kreyenbühl (a. a. O. $205 \mathrm{ff}$ ) freilich soll auch dieser Simon niemals existiert haben. Was vielmehr Josephus von ihm erzählt, seien nichts als Verleumdungen, die die Juden gegen Paulus, von ihnen Simon genannt, erhoben hätten. Aber abgesehen davon, daß auch dieser Hypothese die unrichtige Kombination des Magiers Simon und des Apostels Paulus zu Grunde liegt, so ist gar nicht einzusehen, was Schmiedel ${ }^{x}$ mit Recht hervorhebt, wie Juden dazu gekommen sein sollen, dem Paulus den Namen Simon zu geben. -

Ähnlich hat Krenkel, Josephus und Lukas, Leipzig 1894 I 78 ff. in dem Simon Magus des Josephus den Apostel Paulus gefunden und nur im Unterschiede von Kreyenbühl die. Kuppelei der Drusilla auf judenchristliche Verleumdung zurückgeführt. Aber ist auch diese Hypothese denkbarer als die Kreyenbühls, so beruht doch auch sie auf einer unrichtigen, tendenziösen Auffassung des Apostels Paulus. Überdies mühen sich beide Hypothesen vergeblich ab, eine ausreichende Erklärung dafür zu geben, wie Paulus dazu kam, ein Kúmploc tò révoc zu werden. Denn dazu genügt doch nicht die Erzählung Act $13,6 \mathrm{ff}$. Ebenso lassen es beide, besonders aber Krenkel, unerklärt, wie der jüdische Geschichtsschreiber Josephus darauf verfiel, eine derartige, von jüdischem bezw. judenchristlichem $\mathrm{HaB}$ gegen Paulus erfundene Verleumdung aIs rein geschichtliche Anekdote aufzufassen und so in sein Geschichtswerk aufzunehmen.

I Art. Simon Magus in Encycl. Bibl. p. 4556. 
Wir muissen daher nach wic vor dem Simon des Josephus zugestehen, dah er eine geschichtliche Gröbe ist.

Ist er aber dann nicht derselbe, wie der Simon der Kirchenväter?

Allerdings ist jener Simon ein Jude und Cyprier von Geburt, dieser dagegen ein Samariter aus dem Flecken Gittä, und der Name Simon kommt vcrhältnismäbig so oft vor, dalb mit gutem Grunde beide als zwvei verschiedenc Persönlichkeiten angesehen werden können. ${ }^{3}$ Aber es ist doch auffällig, daß in derselben Zeit und in derselben Gegend zwei Magier gelebt haben sollen, welche beide eine grolie Rolle spielten und Simon hießen; und es ist sehr wohl möglich, daß beide ein und dieselbe Persönlichkeit sind, wie le Moyne, Ittig, Basnage, J. Chr. Wolf, Havercamp, Neander und Simson vermutet haben. ${ }^{2}$ In diesem Falle muß man sich fragen, welcher Nachricht man mehr Glaubwürdigkeit beimessen darf, ob der des Palästinensers Josephus oder der des Samaritaners Justin. Für Josephus spricht, daß er der Zeit nach dem Simon weit näher steht als Justin. $\mathrm{Da}$ er jedoch öfters und gerade in den letzten Büchern seiner Archäologie in seinen Nachrichten recht flüchtig und nicht immer zuiverlässig ist, kann man bei ihm auch leicht einen Irrtum annehmen. Wahrscheinlich hat er durch mündliche Überlieferung die Geschichte von der durch einen Magier Simon vermittelten Heirat des Felix und der Drusilla gehört. Dabei hat er vielleicht vernommen, daß dieser aus Gittä stamme.3 Unbekannt mit dem samaritanischen Flecken Gittä, ${ }^{4}$ hat er dieses Gittä ( $=$ hebr. Gittim) mit der Hauptstadt Cyperns Cittium ( $=$ hebr. Kittim) verwechselts und so den Magier Simon zu einem Cyprier und, da er einen jüdischen Namen führte, zu einem Juden gemacht.. Wird man daher in diesen Angaben über die Heimat Simons dem Josephus einen Irrtum zutrauen können - die übrigen Angaben des Josephus, wie die Heiratsvermittelungsgeschichte, sind gewiß historisch. - , so wird man die ganz bestimmt wiedergegebene Notiz Justins als zuverlässig betrachten müssen, d. h. man wird in dem

I Vgl. Möller Artikel Simon in Herzog R. E.2 XIV 249.

2 Vgl. dazu Simson, das Leben Simons des Magier in Zeitschr. f. hist. Theol. I84r. XI. 3. 20.

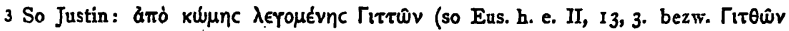
Epiph. haer. 21, 1). Der Ort hieß wahrscheinlich Títral. Vg1. Lipsius die Quellen der römischen Petrussage Kiel I\$72 33 Anm.

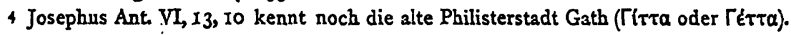

5 Epiph. adv. haer. 30, 25 sagt Ki kai "Pobıol. Nachträglich finde ich dieselbe Annahme bei Hort, Clementine Recognitions London $1901 \mathrm{I} 26 \mathrm{f}$. 
Magier Simon des Josephus und in dem des Justin ein und dieselbe Persönlichkeit erblicken dürfen.

Úber die Zeit dieses Simon würden die Nachrichten des jüdischen Geschichtsschreibers einerseits und die der hier in Betracht kommenden altchristlichen Schriften andrerseits im wesentlichen harmonieren.

Nach Josephus weilte er in Cäsarea zur Zeit des Felix, der 52-60 dort Prokurator war. Da Felix nach Tacitus, Annal. XII, 54; Hist. V, 9, ehe er von Klaudius zum Prokurator von Judäa ernannt wurde, und zwvar schon lange vorher neben seinem Amtsvorgänger Ventidius Cumanus einen Teil der Provinz Samaria verwaltete, kann er sehr wohl bei diesem Aufenthalt den Simon näher kennen gelernt haben, um ihn später als Heiratsvermittler zu benutzen, vorausgesetzt, daß der Simon des Josephus derselbe ist, wie der des Justin.

Wenn alsdann dieser berichtet, $x$ daß er unter Klaudius $(4 \mathrm{I}-54)$ nach Rom gekommen sei, so braucht er sich in dieser ganz bestimmten Zeitangabe nicht deswegen zu irren, weil er sich in der Notiz von der göttlichen Verehrung Simons geirrt hat (s. o. S. 123 Anm. 3). Hat aber Simon dem Felix als Heiratsagent gedient, dann wird er allerdings nicht eher als in den letzten Jahren des Klaudius nach Rom gekommen sein. - Aus dem Berichte der Apostelgeschichte, wie auch aus der oben angeführten Bemerkung des Clemens Alexandrinus läßt sich nicht mehr entnehmen, als daß er ein Zeitgenosse des Apostels Petrus war. Übrigens wissen auch die apokryphen Petrusakten von seinem Auftreten unter Klaudius zu erzählen, ${ }^{2}$ obwohl sie sonst im Widerspruch hiermit den römischen Konflikt des Simon und des Petrus in die Zeit Neros verlegen. Ob sie hierin Justin folgen, wird sich nicht sicher ausmachen lassen.

Gegen diese chronologische Feststellung kann, wie schon Simson (a. a. O. 2 I f.) richtig hervorgehoben hat, die Bemerkung des Hegesipp bei Eus h. e. IV 22, $5^{3}$ nicht, wie von Beausobre geschehen, ins Feld geführt werden, da sie nichts anders besagt, als daß Simon gleichwie Thebuthis aus den jüdischen Sekten hervorgegangen sei.

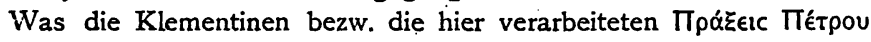
(Rec. 2, 7 Hom. 2, 22. 25) von den Eltern Simons und seiner Erziehung in Alexandrien erzählen, gehört in das Gebiet der Sage, deren etivaige geschichtliche Unterlage nicht mehr festgestellt werden kann. Eben-

I Apol. I, 26. 56. Vgl. Iren. adv. haer. I 23, I. Tert. Apol. r3. Eus. h. e, II, 10 Theodoret haer, fab. I, 1 .

2 Vgl. Act. Petr. c. Sim ed. Lips. et Bonnet I 49.

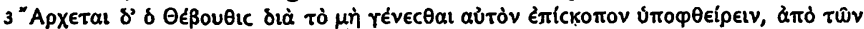

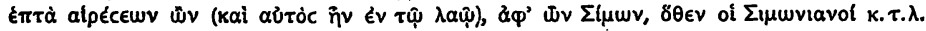


dahin sind illre Angaben von den Beziehungen Simons zu dem Hemerobaptisten Johannes und dessen Schüler Dositheus zu rechnen. Denn sie sind als der sagenhafte Niederschlag einer Geschichtsauffassung zu betrachten, wic sic bei Justin, Hegesipp (bei Eus. h. e. IV, 22, 7), in der

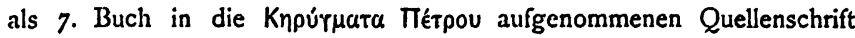
(Rec. I, 54) sowie in den apostolischen Konstitutionen vorliegt, wonach die Johannesjünger (Baptisten bei Justin Dial c. Tryph. 80, Hemerobaptisten bei Hegesipp und Const. ap. VI 8, Johannesjünger Rec. I, 54) und die Dositheaner (bei Hegesipp sowie Const. ap. VI, 8 mit den Simonianern, Rec. I, 54 neben den Samaritanern) $\mathrm{zu}$ den vorchristlichen (jüdischen) bezw. ersten christlichen Häresien gehören und demnach als Vorläufer der Simonianer anzusehen sind. ${ }^{x}$

Über die Wirksamkeit Simons enthält die Apostelgeschichte (Act. 8, 9-24) den ältesten Bericht. Danach hat er in Samarien magische Künste getrieben und durch seine Wunder die Menge in Staunen gesetzt (8, 9. II). Dasselbe berichtet Justin, Apol. I, 26. 56, indem er seine magischen Künste als Werke der Dämonen ansieht. Ihm folgt Iren. adv. haer. I 23, I. Damit stimmt nicht nur die allgemein übliche Bezeichnung Simons als eines Magiers, sondern auch die Nachricht des Irenäus und Tertullian, sowie der Schrift de rebaptismate (s. o.), daß seine Anhänger Zauberei, insbesondere Totenbeschwörungen betrieben.

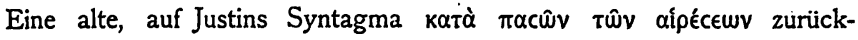
gehende Quelle, die im Carm. adv. Marc. I I57-I 59 erhalten ist, ${ }^{2}$ erwähnt als Beweise seiner magischen Verblendung, daß er überall hindurch wandern und fliegen könne. Eine ähnliche Überlieferung liegt bei Arnobius adv. gentes İं, I 2 vor, wonach Simon in Rom auf einem Wagen mit einem feurigen Viergespann gen Himmel zu fahren versucht habe. Auf einen Flugversuch Simons deutet auch Epiphan, haer. 2I, 5 hin.

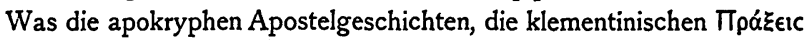
Tét $\operatorname{lov}^{3}$ sowie die späteren Petrusakten 4 davon erzählen, harmoniert mit

I Die Hinterordnung der Dositheaner hinter die Simonianer bei Hegesipp (Eus. IV, 22) bedeutet nicht eine zeitliche Nachordnung, da ja dann Hegesipp, wie Hilgenfeld, Judentum und Judenchristentum Leipzig 1886, S. 42 Anm. 80 richtig bemerkt, die von ihm an derselben Stelle noch hinter die Dositheaner gestellten Masbotheer zeitlich erst nach Simon und Dositheus gerechnet haben mübte, während er sie doch schon zu den 7 jüdischen Stammhäresien zählt (Eus. h. e. IV, 22, 7), hier neben den Hemerobaptisten.

2 Vgl. meine Schrift, Das pseudotert. Gedicht adv. Marcionem Darmstadt 1901, S. VII.

3 Vgl. Rec. 2, 9; 3, 47.48.60. Hom. 2, 32. 34; 3,30; 4, 4 .

4 Vgl. Act. Petr. c. Sim 1. c. 6r. 7I Act. Petr. et Paul. 1. c. 194. 
den angeführten patristischen Zeugnissen; zeigt jedoch durchgängig eine Weiterbildung der Legende. Offenbar übertrug man auf Simon, der als Magier bekannt war, allerlei von dem, was man sich damals von andern Goëten wie z. B. Apollonius von Tyana u. a. zu erzählen wußte.

Seine magische Kunst, vielleicht auch sein dadurch vermittelter Umgang mit dem römischen Prokurator von Samarien, Felix, verliehen ihm unter den Samaritanern ein derartiges Ansehen daß er den Anspruch erheben konnte, ein großer zu sein (Act 8,9 ). Dieses wieder bildete die Voraussetzung für die Bezeichnung, die ihm das samaritanische Volk

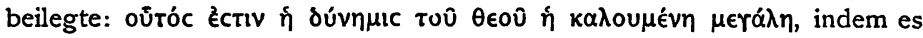
ihn geradezu als ein höheres Wesen, einen Engel verehrte. ${ }^{x} \mathrm{Daß}$ er der Magier, mit dem Evangelisten Philippus anknüpfte, während dieser in Samarien wirkte, Kranke und Dämonische heilte (Act 8, I f. I 3 ff.), wird nicht von vornherein deswegen für völlig unhistorisch angesehen werden müssen, weil der Bericht der Apostelgeschichte überhaupt nur in später überarbeiteter Gestalt vorliegt. ${ }^{2}$ Vielmehr wird èr insoweit als zuverlässig gelten dürfen, als an Stelle des Philippus der Apostel Petrus eingesetzt wird. Ein Zusammentreffen des Petrus und Simon (in Samarien bezw. Syrien) setzten ja auch nicht nur Klemens von Alexandrien, sondern auch die Petrusakten voraus. ${ }^{3}$

$\mathrm{Da}$ ß Simon sich später in Cäsarea Stratonis, der Hauptstadt Palästinas, aufhielt, scheint daraus hervorzugehen, daß Josephus ihn als Heiratsvermittler des Felix auftreten läßt; denn diese Geschichte ist wohl deshalb erst in die Zeit, wo Felix in Cäsarea residierte, zu verlegen, weil er schon früher verheiratet war und auch erst als oberster römischer Beamter der Provinz es wagen konnte, eine herodianische Prinzessin zu veranlassen, ihren königlichen Gemahl verließ und ihn heiratete.

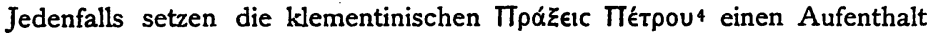
des Simon in Cäsarea voraus (ebenso die späteren Petrusakten).s Wenn sie ihn von Petrus durch Syrien bis nach Antiochien verfolgt werden

I Es ist jedoch möglich, daß dieser Ausdruck auch im gnostischen Sinne zu ver-

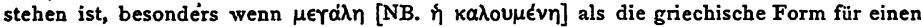
aramäisches Wort wie מנלא (- Offenbarer) zu fassen ist, gvl. Klostermann, Probleme im Aposteltext 188315 ff. nach Schmiedel Art. Simon Magus in Encycl. Bibl. p. $455^{8}$.

2 Vgl. besonders die Notiz von dem Vorrecht der Apostel, den hl. Geist durch Handauflegung mitzuteilen.

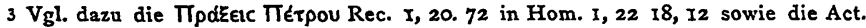
Petr. c. Sim. 1. c. 49. 50. 54. 56.63. 65. 71 sowie meine Untersuchung in T. u. U. N. F. X, 4. S. $227 \mathrm{ff}$.

4 Rec.. I, 72.74 3, 63 Hom. I, 15 4, 2 6, 26, vgl. Const. Ap. VI 8.

5 Vgl. Act. Petr. c. Sim. 1. c. 49. 50. Marc. Petr. et Paul. 1. c. 142 cf. 200. 
und dann wieder nach Judäa (Cäsarea?) fliehen lassen, so knüpfen sie an die Erzählung der Apostelgeschichte an, wie sie diese in dem Bericht von der mehrmaligen öffentlichen Beichte Simons voraussetzen. Wenn sie ihn als Magier und große Kraft Gottes bezeichnen?, so lassen sie dabei an einzeinen Stellen gnostische Vorstellungen mit unterfliefien.2 Aber im allgemeinen ist doch Simon auch hier derselbe, als der er in Act. 8 dargestellt wird. Auch darin findet sich eine gewisse Ähnlichkeit, daß sie $^{3}$ ihn - in dem eigentlichen Reisebericht wenigstens - stets ohne Helena auftreten lassen. Über Act. 8 auf eine ältere Überlieferung weisen sie zurück, wenn sie als seinen Gegner nur Petrus - und nie Philippus bezw. Johannes - nennen.

Wenn Justin den Simon schließlich nach Rom kommen läßt, so folgt er damit einer Überlieferung, welche dieselbe Glaubwürdigkeit besitzt wie seine chronologische Angabe. Nun aber hat sich uns bereits diese Zeitbestimmung (Klaudius) durch Vergleich mit andern Nachrichten als richtig erwiesen. So wird sie, wie sie auch durch die klementinischen

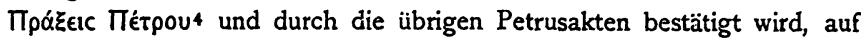
eine Tradition zurückgehen, deren Träger die auch in Rom vorhandenen Simonianer gewesen sein mögen. Die Erzählungen dagegen von seinem Ende in Rom beruhen auf späterer Erfindung. Die Fabel von seinem milglückten Flugversuch geht vielleicht auf die Erzählung des Sueton von dem Ende eines Gauklers zur Zeit Neros zurück.s Was Hippolyt Phil. VI, 20 berichtet - sein freiwilliges Begräbnis - ist nichts als ein Gegenstïck zur Grablegung und Auferstehung Christi.

Auf einer späteren Kombination der Simon-Petruslegende und der Petrusgeschichte beruht jedenfalls die Angabe, wie wir sie in den apokryphen Apostelgeschichten sowie bei späteren Kirchenvätern finden, $\mathrm{da} B$ er in Rom unter Nero geendet habe. Sicher hat auf diese Tradition, die mit der durch Justin, Irenäus, Euseb und Theodoret bezeugten, die nur die Zeit des Klaudius kennt, im Widerspruch steht, sowohl die Erzählung des Sueton als auch die Überlieferung von dem Martyrium des Petrus zur Zeit der neronischen Christenverfolgung bestimmend eingewirkt.

Schweben aber auch diese Angaben über das Ende Simons in der Luft, so ist als ein fester Bestandteil der Überlieferung anzusehen, daß Simon mit Petrus in Berührung gekommen ist. $\mathrm{Da}$ jedoch beide

I Vgl. Rec. 1,72 2, 7 Hom. 2, 22 cf. 3, 2 18, 4, Act. Petr. c. Sim. l. c. 48.54 Martyrium Petr. et Paul. 132. I44. 156.

3 So auch die'späteren Petrusakten.

2 Vgl. Hom. 3, 2 18, 12.

5 Vgl. Sueton Nero 12.

4 Vgl. Rec. 3,63. 
sich auch in Rom gegenübergetreten sind, wie in Samarien, kann nicht als historisch bezeugt gelten. Wenn Simon unter Klaudius, Petrus aber erst unter Nero nach Rom gekommen ist, dann können beide nicht, wie uns dies auch das Schweigen des Justin und Irenäus bestätigt, in Rom mit einander gekämpft haben. Und es war erst die zum Teil legendarische Fortbildung jener geschichtlichen Überlieferung, wie sie in den - auch in den Klementinen verarbeiteten - Petrusakten vorliegt, welche den Kampf zwischen Simon und Petrus, dessen ursprünglicher Schauplatz Samarien war (Act. 8), über ganz Syrien ausdehnte und nach Rom verlegte. Erst auf Grund hiervon konnte die Nachricht aufkommen, die als ältestes patristisches Zeugnis für eine gemeinsame Anwesenheit des Simon und Petrus in Rom zu gelten hat, daf Petrus eine Disputation mit Simon auch in Rom unter einer Platane gehabt habe, infolge deren Simon ein trauriges Ende fand.

Eine noch spätere Überlieferung hat mit dem Ende des Simon auch das des Petrus verbunden:

II.

Dieses Simonbild der Geschichte wurde jedoch nicht nur in sagenhafter Weise' ausgeschmückt, sondern nahm auch im Laufe der Zeit unter dem Einfluß neu hinzutretender religiöser und philosophischer Ideen ein yöllig andersartiges Gepräge an. Diese Entwicklung erfolgte zunächst, wie es scheint gleichzeitig, in einer doppelten Richtung, auf samaritanischsyrischem Boden in mythologischer, auf alexandrinischem Boden in philosophischer Richtung.

Beidemal geht sie jedoch von dem historischen Simonbild aus.

Versuchen wir nun diese Entwicklung darzustellen, so haben wir an die volkstümliche Verehrung anzuknüpfen, welche Simon in Samarien genof. Wurde er hier, wie die Apostelgeschichte berichtet, von dem Volk als die große Dynamis, d. h. als ein himmlisches Geistwesen verehrt, was lag da im Kreise seiner Anhänger näher, als ihn selber deri Anspruch -erheben zu lassen, er sei die große Dynamis Gottes, ja Gott selbst? So erzählt bereits Justin, Apol. I, 26. 56, daß fast alle Samaritaner ihn als den obersten Gott verehrt hätten, der über allen Engeln stehe. Dementsprechend gibt Irenäus adv. haer I, 23. i. die Behauptung Simons wieder, esse autem se sublimissimam virtutem, hoc est eum, qui sit super omnia pater; ebenso Pseudotertullian adv. omn. haer. I. hic ausus est summam se dicere virtutem, id est summum deum; Epi-

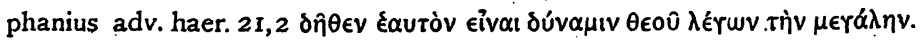




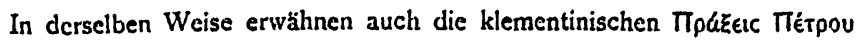

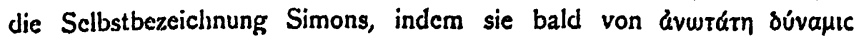
(Hom. 2, 22), summa bezw. prima virtus (Rec. I, 72 2, 12 3, 47) reden, bald diese mit dem obersten Gott identifizieren; vgl. $\theta$ eòc avútatoc (Hom. 2, 22 3. 2) excelsus deus (Rec. I, 72 cf. 2, 7) summus deus (Rec. 2, 13).2 Tritt nun aber diese Identifizierung Simons mit dem obersten Gott zuerst bei Justin auf, so muß sie bereits vorher erfolgt sein. Nun erscheint es bedeutsam, dafi im engsten Zusammenhang mit dieser Notiz bei Justin zum ersten Male der Helenamythus erwähnt wird, der in der kanonischen Apostclgeschichte, wie auch in den apokryphen fehlt und in den Petrusakten der Klementinen erst später hinzugefügt worden ist. In dem Berichte von den Kämpfen des Simon und Petrus wird er wenigstens nirgends erwähnt, außer in der Einleitung (Rec. 2 Hom. 2), wo auch (s. o.) sonstiges legendarisches Material z. B. über den Vorläufer Simons, Dositheus verwertet ist. Erst Justin Ap. I, 26 (vgl. Eus. h. e. II, I3) erzählt, daL die Samaritaner neben Simon als dem obersten Gott auch die Helena, die früher in einem Bordell in Tyrus gelebt habe, als seine erste Ennoia verehrt hätten. Dasselbe wie Justin berichten auch die alte Quelle im Carm. adv. Marc. I, I59, Irenäus I, 23.2, der sie wie Tertullian de ania 34 nach Tyrus versetzt, Hom. 2, 23. 25 18, 12 Rec. 2, 9. I 2 die sie allerdings zuerst mit Dositheus (in Alexandrien) in Verbindung bringen, aber doch auch von ihrem Verhältnis zu Simon zu erzählen wissen (Rec. 2, 12).

Wie ist es möglich, daß seit Justin neben Simon diese Helena er-

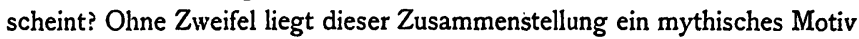
zu Grunde, wie es zuerst Baur (Manich. Religionssystem 467f.) erkannt hat. ${ }^{2}$ Werden wir nämlich mit der Helenageschichte von Samarien auf einmal nach Tyrus versetzt, so können wir uns diese Entwickelung nicht anders vorstellen, als daß sich die samaritanische Verehrung Simons als des obersten Gottes mit der phönizischen, speziell tyrischen Verehrung des Sonnengottes (Sem, Schemesch, Herakles, Melkart, Baal) und der Mondgöttin (Helena, Selene, Luna, Astarte) verbunden hat. Ob zu dieser Verbindung die Ausbreitung des Simonkultus über die Grenzen Samariens nach Phönizien, oder umgekehrt der Einfluß des phönizischen Sonnenund Mondkultus auf die samaritanische Volksreligion den Anlaß gegeben hat, wird sich schwer ausmachen lassen. Nicht unmöglich erscheint

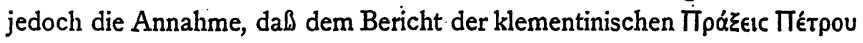

I Beide Ausdrücke scheinen auf eine gemeinsame hebräische Wurzel zurückzugehen,

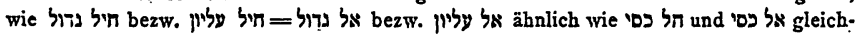
gesetzt wurde.

2 Vgl. Lipsius, Apokryphe Apostelgeschichten II. I, $34 \mathrm{ff}$. 
von der Verfolgung Simons durch Petrus durch die phönizischen Küstenstädte von Cäsarea über Sidon-Tyrus nach Antiochien der geschichtliche Sachverhalt zu Grunde liegt, daß gerade hier der Simonkultus Einflu@ gewonnen hatte, bis er durch die Predigt des Evangeliums verdrängt wurde. Ein andres Motiv für diese Lokalisierung der Sagenbildung wird sich schwerlich ausfindig machen lassen. Jedenfalls muß diese Verschmelzung des Simonkultus mit der syrischen Volksreligion noch um die Wende des I. und 2. Jahrhunderts erfolgt sein, da Justin sie kennt, ohne zu verraten, daß er diesen Zusammenhang durchschaue.

Weiter nichts als eine christliche Deutung der verschiedenartigen Bedeutung, welche Simon als samaritanischer Messias und syrischer Volksgott hatte, ist die Auffassung, welche sich zuerst bei Iren. adv. haer. I, 23. I findet, daß er den Juden als Sohn (= Messias) erschienen ${ }^{3}$ in Samarien als Vater (= oberster Gott) herabgestiegen und zu den übrigen Völkern als heiliger Geist (= Helena) gekommen sei (vgl. Theodoret haer. fab. I, I). Eine Umbiegung dieser irenäischën Auffassung ist es, wenn Cyrill Cat..VI, I4 und Augustin de haer. I berichten, daß er in der Person des Vaters auf dem Sinai den Juden das Gesetz gegeben, (zur Zeit des Tiberius) in einem Scheinkörper als Sohn sich gezeigt und später als heiliger Geist in feurigen Zungen sich über die Apostel ausgegossen habe.

\section{III.}

Also zum Gott geworden, mußte sich aber Simon bald eine neue Metamorphose gefallen lassen. Wie überhaupt seit dem beginnenden Verfall der heidnischen Volksreligionen, besonders in den Kreisen der Gebildeten, die volkstümlichen Gottheiten, mit dem Mittel der Allegorie in philosophische Ideen aufgelöst wurden, so wurden nun auch Simon und Helena spiritualistisch umgedeutet. So entstand jenes gnostische System, das als das simonianische bezeichnet wird, von Simon jedoch kaum mehr als seinen Namen hat. Seine Grundzüge sind bereits bei Justin angedeutet, aber bei. den späteren, auf Justin beruhenden Häreseologen und

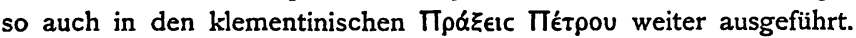

Danach ist Simon nach wie vor die erste Dynamis, die oberste Gottheit, die jedoch jetzt philosophisch als der ewige und letztlich unbekannte Urgrund des Weltlebens gedacht wird (Justin; Carm. adv. Marc.

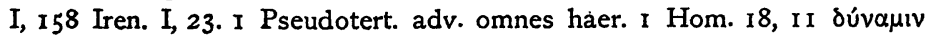

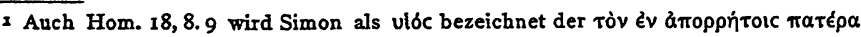
offenbart. 


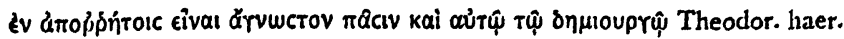

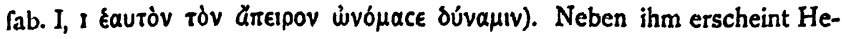
lena als seine erste Ennoia, so schon bei Justin Apol I, 26. Sie ist die Erstgeborne Gottes, die Mutter der gesammten Geisterwelt, wie sie auch Herrin (kupi $\alpha$ ) Allmutter ( $\pi \alpha \mu \mu \eta \tilde{r} \omega \rho)$ und Weisheit (copí $\alpha$ ) genannt wird (Hom. 2, 25 18, 12 cf. Rec. 2, 2), Iren.: prima mentis ejus conceptio ennoia; Tert.: injectio sua prima, Iren.: mater omnium). Auch heiliger Geist ( $\pi v \in \hat{u} \mu \alpha$ árıv) heillt sie nach Epiph. haer. 21. 2 in dem Sinne, wie bei den jüdischen Theosophen nin die schöpferische Lebenskraft der Welt bedeutet. Doch ist ihr dieser Name erst später beigelegt worden, ebenso die Bezeich-

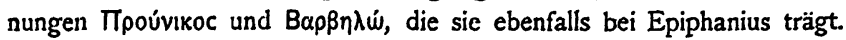
Offenbar hat man in Folge einer leichtverständlichen Ideenassociation die gnostischen Bezeichnungen der Sophia auf die simonianische Helena übertragen.

Durch sie hat Simon die Engel und Erzengel geschafien (Iren. adv. haer. I, 23, 2. Tert. de anim. 34. Hom. 18, 12 Pseudotert. adv. omn. haer. I. Epiphan. adv. haer. 2I, 2, Theodor. haer. fab. I, I), denen er nach Epiphan. haer. 2I, 4 barbarische d. h. orientalische Namen beilegte. Durch diese Engel, welche ihren Vater nicht kannten (Hom. I8. II Iren. I, 23, 2 Tert. de anim. 34) ist die Welt gegrundet worden (vgl. noch Epiph. haer. 22, 2); durch sie sind auch die Menschen gebildet, die Gesetze gegeben und die Propheten inspiriert worden (vgl. Hom. 3, 2 18, 12 Hippolyt Philos. VI, ig Epiph. haer. 2I, 4 Theodoret haer. fab. I, r), um die Menschen in Knechtschaft $z u$ halten. Als aber die Engel so durch die Helena-Ennoia zwar nicht wider Wissen und Willen des Vaters, aber doch vor seinem Entschlu@ geschaffen worden waren, hielten sie ihre Mutter bei sich zuruick, um nicht als unselbständige Kreaturen gelten zu müssen, wehrten ihr die Rückkehr zum Vater, schlossen sie in weibliche Körper ein und zwangen sie so von einem Körper zum andern zu wandern (a. a. O.). So ist sie in der Person der griechischen Helena erschienen, welche den Trojanischen Krieg veranlabt und den Stesichorus seiner Schmähgedichte wegen mit Blindheit geschlagen und erst auf seinen Widerruf wieder sehend gemacht hat (Iren. I, 23, 2 Tert. de anim. 34 . Hom. 2, 25 Rec. 2, 2 Epiph. haer. 21, 3 Theodor. haer. fab. I, 1), wie schon die griechischen Philosophen die Helena auf Grund von Hom. Od. IV, 121. I22 in ähnlicher Weise allegorisch deuteten (vgl. Eusthatius Commentar in Hom. Od. IV, I2I). Wenn die klementinischen

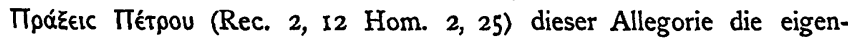
artige Wendung geben, daß die Griechen und Trojaner nur das Bild 
der Helena-Ennoia gesehen hätten, während sie selbst bei dem obersten Gott weilte, so haben sie diesen Gedanken ebenfalls der griechischen Philosophie entnommen. (Vgl. Plato de republ. IX p. 586 ed Steph. Sext. Empiricus adv. mathem. VII p. 407 ed. Fabricius; Aristides, Orat. Platonic. I pro rhetorica p. 92 ed Canter; Euripides Helena 31 sq bei Simson a. a. 0.64 Anm. 65). Zuletzt ist Helena in den Körper jener tyrischen Hure gewandert. $\mathrm{Da}$ aber ist in Simon der oberste . Vater selber vom Himmel herabgestiegen; um sie, das verlorne Schaf, zu befreien: Um jedoch den Nachstellungen der Engel zu entgehen, hat er sich den verschiedenen Himmelsregionen angepabt. So ist er, den Engeln unbekannt,. bis auf die Erde herabgekommen, als Mensch unter Menschen erschienen und hat scheinbar in Judäa gelitten. Auf diese Weise hat er die Ennoia von der Macht der Engel befreit, den Menschen aber durch seine Erkenntnis das Heil zugesichert (Rec. 2, I4 3, 47 Hippolyt Philos VI, 19 Cyrill Cat. 6, 14 Iren. I, .23. 3 Tert de anim. 34 Pseudotert. adv. omnes haer. 1 Epiphan. haer. 2r,2. 3 Theodoret haer. fab. I, I Philastr. 29).

Der gnostische Charákter dieses Systems ist unverkennbar, zumal in seiner Verwandtschaft mit der valentinianischen und ophitischen Gnosis. Und man wird Justin Recht geben, wenn er den Simon; der dieses gnostische System erfunden haben soll, in eine Reihe mit den übrigen Gnostikern, ja an ihre Spitze stellt. Denn vergleicht man es mit den genannten gnostischen Systemen, so wird màn es auch als das einfachere und darum primäre bezeichnen müssen.

Aber das ist ebenso unverkennbar, da $\mathrm{der}$ Simon dieses gnostischen Systems nicht selbst Gnostiker, sondern die Verkörperung der göttlichen Idee selber ist. In dieser Bedeutung ist er derselbe geblieben, der er in dem samaritanischen Kult geworden war. Alles andre ist gnostische Zutat.

Die Heimat dieses Systems ist Syrien. Nur hier konnte man däran denken, Personen. kultischer Verehrung, wie den Simon = Sonnengott und die Helena = Mondgöttin, zu dein Hauptakteuren eines gnostischen Welterlösungsdramas zu machen. Auf Syrien weist auch die Stelle, die dabei den Engeln und Erzengeln zugewiesen ist. Justin kennt schon die Grundzüge dieses Systems, das er in Syrien kennen gelernt haben wird. Es muß also vor ihm entstanden sein. Wenn nach Iren. I, 23, 4, dem Hippolyt Phil. VI, 19; Epiphan. haer. 21, 3 Theodoret haer. I, I Augustin de haer. I Eus. h. e. II, 13, 6 folgen, die Simonianer den Simon unter dem Bilde des Jupiter, die Helena unter dem Bilde der Minerva verehrten, so sind sie die Träger dieses gnostischen Systems gewesen. Denn unter dem Bilde der Minerva konnten sie nur dann jene Helená 
verehren, wenn sie sie nicht mehr als Mondgöttin kannten, - daraus hätten sic eine Artemis-Diana machen müssen -, sondern wenn sie in ihr bereits die Personifikation der göttlichen Weisheit, die Ennoia des gnostischen Systems, sahen.

\section{IV.}

Wie auf syrischem Boden sich das gnostische System des Simonianismus aus dem samaritanisch-syrischen Simon- und Helenakult und dieser wiederum aus der abergläubischen Verehrung des historischen Simon Magus entwickelte, in ähnlicher Weise, jedoch in einer anderen Richtung, bildete sich der Simonianismus auch in Alexandrien aus.

Auch hier knüpfte er an die Bezeichnung Simons als der großen Dynamis Gottes an, wobei Simon entweder als der Offenbarer.dieser Dynamis, also als ein Messias galt, oder aber mit ihr selbst gleichgesetzt wurde. Nach einer zweifachen Richtung aḅer entwickelte er sich im Unterschied von dem samaritanisch-syrischen Simonianismus. Einmal fehit ihm vollständig das mythische Element, wie es besonders in dem Helenamythus zum Ausdruck kommt; an seine Stelle tritt vielmehr ein philosophisches Element, das dem alexandrinischen Hellenismus entnommen ist. Auf dieser Grundlage entfaltet sich sodann das gnostische Element in einer philosophisch-esoterischen Richtung, welche dem simonianisch-gnostischen System des Justin-Irenäus durchaus fehlt.

Skizzieren wir nun diese Entwickelung, so finden wir zunächst die auffallige Tatsache, dab in den Nachrichten über Simon, die uns nach Alexandrien weisen, der Helenamythus fehlt. Clemens Alexandrinus nennt ihn nicht, und auch Origenes scheint ihn nicht gekannt zu haben. Denn wenn Celsus, (bei Orig. c. Cels. V, 62), erwähnt, daß die Simonianer auch Helenianer hießen, weil sie einer Helena oder einem Helenus, der ihr Lehrer gewesen sei, Verehrung erwiesen hätten, so hat er zwar von der simonianischen Helena etwas gehört, bringt sie aber, weil er nichts damit anzufangen weib, mit dem im Altertum berühmten Weissager Helenus zusammen. Origenes aber hat, wie es scheint, auch nicht viel mehr davon gewußt wie Celsus. Denn während er wohl auf den Vorwurf des Celsus, daß die Christen auch Simonianer hießen, zu erwidern wei $\emptyset$, die Simonianer erkennten nicht Jesus als den Sohn Gottes, sondern Simon als die Kraft Gottes an, geht er über die Unkenntnis oder das Mißverständnis des Celsus hinsichtlich der Helena mit Stillschweigen hinweg - ein Anzeichen dafür, da $\mathrm{B}$ auch er von der Helena nichts wußte. 
Während also Helena hier fehlt, erhält Simon selbst, als die Kraft Gottes, eine Bezeichnung, die Justin und die ihm folgenden Häreseologen nicht kennen, den Namen Écrúc. Zum ersten Mal stoßen wir auf diesen Namen bei Clemens Alexandrinus, der Strom II, 1 I, 52 bemerkt:

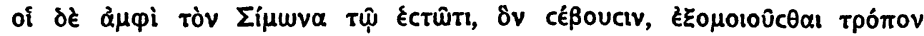
ßoúlovtal. Ebenso finden wir ihn auch in den klementinischen

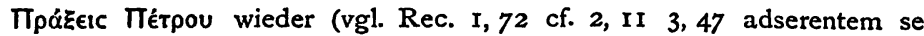
esse quendam stantem, hoc est alio nomine, Christum; 2, 7 et Christum putari atque stantem nominari. Hom. 2, 22 évióte $\delta E$ kà̀ Xpıc-

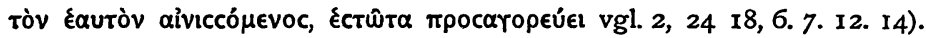
Beachten wir nun, daß die Klementinen gerade in diesen Zusammenhang (wenigstens in Hom. 2, 22) in auffalligem Widerspruch mit der übrigen Tradition erzählen, daß Simon seine Erziehung in Alexandrien genossen habe und in Alexandrien zu der Würde des 'Ectúc gelangt sei, so werden wir hierin ein weiteres Anzeichen dafür finden dürfen, $\mathrm{da} B$ gerade in Alexandrien ihm dieser Name beigelegt wurde. Darauf weist aber auch, daß dieser eigentümliche Ausdruck gerade in der alexandrinischen Philosophie gebräuchlich ist. Bei Philo steht er wiederholt, u. zw. als Beinamen des höchsten Wesens, wenn er schreibt de nominum

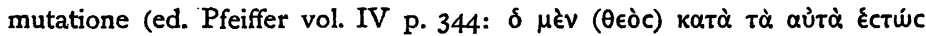

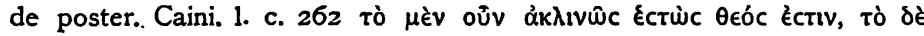

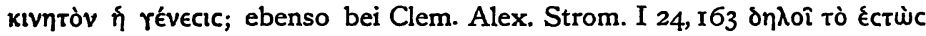

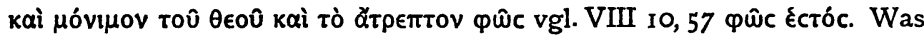
nun die Bedeutung dieses Ausdruckes anlangt, so sieht Rec. I, 72 ebenso Hom. 2, 22 darin eine andre Bezeichnung für Christus. In gleicher Weise wird Hom. 18, 6. 7. 2. 14 der Ausdruck Éctẃc mit dem Ausdruck víóc gleichgesetzt. Es haben sich infolgedessen viele Ausleger, wie z. B. Ritschl, dazu verleiten lassen, in dieser Bezeichnung einen Beweis für das Pseudomessiastum Simons zu erblicken. Erwägt man

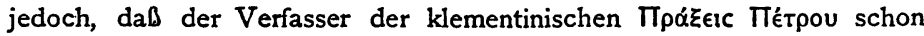
darin irrt, wenn er hierin eine Selbstbezeichnung Simons findet, so wird man ihm uiberhaupt nicht viel Verständnis dafür zutrauen. Dieser Verdacht wird dadurch verstärkt, daß er überhaupt nichts rechtes mit dieser Bezeichnung anzufangen weiß. So erklärt er zwar Rec. 2, 7: Hac autem appellatione utitur, quasi qui neget posse se aliquando dissolvi, adserens carnem suam ita divinitatis suae virtute compactam, ut possit in aeternum durare. Hinc ergo stans appellatur, tanquam qui non possit ulla corruptione decidere (cf. 2, I I 3,47). Zugleich bringt er aber diese Erklärung in eine Verbindung mit der Legende von dem Sturze 
des Dosithcus, der vorher die Würde eincs écrúc getragen habe und in dernselben Augenblick, als er ihrer durch Simon beraubt wurde, gestorben sei; denn er war ja da nicht mehr der éctẃc.

War er daher auch im Besitz einer richtigen Erklärung, so besab er doch dafür nicht das richtige Verständnis.

Dieses gewinnen wir erst, wenn wir uns an die philonische Bezeichnung Gottes als des éctúc halten. Danach ist er nicht ein andrer Ausdruck für den Messias, sondern für Gott selbst. In diesem Sinn ist auch Simon, als die große Kraft Gottes, ähnlich wie in Samarien, auch hier in Alexandrien als Gott selbst, genauer, als das unveränderliche Urwesen der Welt, das unvergänglich und ewig ist, betrachtet worden; vgl. Rec. 2, 7. Der Pseudomessias, der früher ein Zauberer war, ist Gott geworden, aber nicht wie in Samarien in mythischem, sondern in philosophischem Sinne.

Diese alexandrinische Ausgestaltung des Simonianismus ist nun aber auch noch nicht das letzte Glied der Entwicklung überhaupt. Wie viel mehr an jenen samaritanisch-syrischen $Z$ weig, so setzt sich auch an diesen alexandrinischen ein gnostisches System an, das, von jenem wesentlich verschieden, nur unter der Voraussetżung eines alexandrinischen Simonianismus verständlich ist, wie wir ihn gekennzeichnet haben; es ist das gnostische System, welches Hippolyt, Philos. VI, 7-18 auf Grund eines bei den Simonianern in kanonischen Ansehen stehenden Buches,

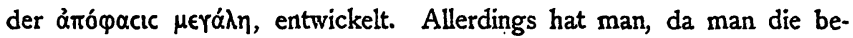
deutende Differenz zwischen diesem gnostischen System und dem, welches aus der Schilderung der übrigen Häreseologen bekannt ist, sich nicht erklären konnte, die Behauptung aufgestellt, daß Hippolyt, der auch sonst nicht ganz schwindelfrei sei, sich dieses System der ámópacic $\mu \in \gamma a ́ \lambda \eta$ gleichsam aus den Fingern gesogen habe. Ehe man aber ein System, wie es Hippolyt entwickelt, als eine Erfindung betrachtet, wird man doch zuvor sich fragen müssen, ob es nicht existiert hat. Und unsre Untersuchung wird uns davon überzeugen. Denn abgesehen davon, daß auch Theodoret, haer. fab. I, I dieses System kennt, so zeigt es in der Schilderung des Hippolyt dieselben Grundzüge, die wir auch bei

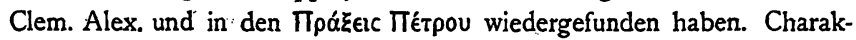
teristisch ist nämlich auch hier ein doppeltes. Vor allem ist auch hier nirgends auf den Helenamythus Bezug genommen, sodaß nicht einmal der Name Helena oder Ěvvoı vorkommt. Sodann tritt auch hier die

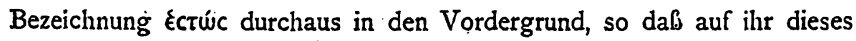
ganze System geradezu basiert ist. Dabei wird der éctẃc in derselben 
Weise aufgefaßt, wie bei Philo die Gottheit. Der verborgene Urgrund aller Dinge ist die unbegrenzte Dynamis, die auch in stoischer Weise - und das mag auf Rechnung Hippolyts kommen - als Feuer bezeichnet wird. Dadurch, daß er nun aus dem Verborgenen in die Sichtbarkeit übergeht, bringt er zunächst aus sich die materiellen treibenden Kräfte der Welt in

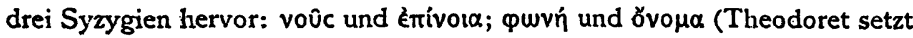

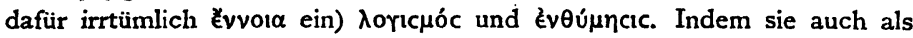
Himmel und Erde, Sonne und Mond, Licht und Wasser bezeichnet werden, stellen sie zugleich die einzelnen Stadien des Weltprozesses dar, in dem der Urgrund der Dinge zu seiner Selbstentwicklung kommt. Wird nun die Dynamis aller Dinge in ihrem An und für sich sein als éctúc bezeichnet, insofern sie von aller Entwicklung unberührt ist, so heißt sie cróc insofern sie in den genannten Syzygien in den Fluß der Dinge eingegangen ist; crncó $\mu \in$ voc dagegen wird sie genannt, insofern sie das Ziel ihrer Selbstentwicklung, ihre Selbstvollendung, findet. Das aber geschieht in dem Menschen, in dem die göttliche Dynamis zu ihrer Ausbildung gelangen soll Auf dieselbe Weise ist. Simon Gott geworden, - wie die Dynamis zu ihrer-Selbstvollendung gelangt. Es leuchtet ein, dab in diesem

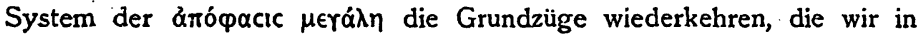
den alexandrinischen Nachrichten über Simon fanden: Umgekehrt finden diese ihre Erklärung in dem System der ảmópacıc. Wenn nämlich die

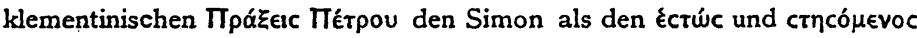
dét bezeichnen, weil er durch seine göttliche Dynamis die Unvergänglichkeit besitze, so wird dies nicht, wie sie es tun, durch eine Legende zu erklären sein, sondern durch die Grundgedanken des gnostischen Systems, wie es Hippolyt darstellt. Ebenso findet hier die sonst dunkle Stelle bei Clem. Alex. Strom. II, I I, 52 ihre Erklärung, wonach die Simonianer es als ihr Höchstes betrachten "dem Ectúc gleich zu werden. Das kann nichts anders bedeuten; als $d a b$ auch sie den Entwicklungsprozeß zu ihrer Selbsterlösung in sich darstellen wollen, durch den die oberste Dynamis zu ihrer Selbstvollendung gekommen und Simon zu Gott geworden ist. Auch sie, die noch ctávtec sind, d. h. im Fluß der Entwickelung stehen, wollen crncó $\mu \in v o l$ werden, d. h. in die selige und unbegrenzte Dynamis aufgehen, in der die Gottheit ihre Selbstvollendung

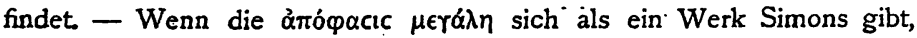
so ist sie eine Fälschung, die aus dem alexandrinischen $Z$ weig der simonianischen Secte hervorgegangen ist. Sicher ist sie jünger als

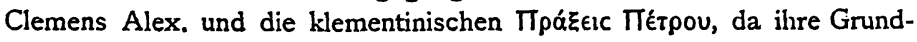

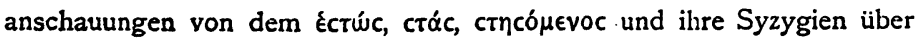


die Anschauungen hinausgehen, die wir in den Klementinen und auch sonst Hippolyt vorfinden. Wenn Hieronymus im Kommentar in Matth 24, als Behauptung Simons die Worte anführt: ego sum sermo dei, ego sum speciosus, ego Paracletus, ego omnipotens, ego omnia dei, so wird als das zunächst liegende angenommen werden dürfen, dab sie auch der ámópacic $\mu \in \gamma \alpha \dot{\lambda} \eta \eta$ entstammen. Auch der Ausdruck sermo dei würde uns nach Alexandrien führen, als dem Orte, wo dieses System entstanden ist und von wo es nach Rom zur Zeit Hippolyts gebracht worden ist.

Von einem Zusammenhang dieses alexandrinischen Systems mit dem samaritanisch-syrischen wird nicht die Rede sein können, außer daß sie - als zwei verschiedene Äste - aus einem Stamm hervorgegangen sind; und es erscheint als eine gekünstelte Konstruktion, wenn Möller in Herzog RE2 XIV S. $255 \mathrm{f}$ jenes aus diesem hervorwachsen läßt.

So sind beide gnostische Systeme mehr oder weniger selbständige philosophische Umdeutungen eines samaritanischen Kultes, der an die Person eines Magiers Simon anknüpfte. $\mathrm{Da}$ dieser Kult eine Universalreligion gewesen sei, wie Harnack, Dogmengeschichte I' ${ }^{1}$ I79. 240 vermutet, müssen wir allerdings in Abrede stellen, da es sich dabei höchstens um eine samaritanische Volksreligion handelt, deren Propaganda kaum über Syrien hinausgekommen ist. Aber in den mehr oder weniger zahlreichen Kreisen seiner Anhänger weiter gepflegt, ist dieser Kult in Syrien unter griechischem, in Alexandrien unter jüdisch-hellenistischem Einfluß zu einer gnostischen Schulmeinung verkümmert, in analoger Weise, wie auch das Christentum in gnostischen Schulen umgeprägt und also weiter überliefert wurde.

Ohne jede innere Berührung mit christlichen Ideen stellt doch diese Entwickelung des Simonianismus eine historische Parallele dar zur Entwicklung des Christentums von seiner Urzeit bis zur Zeit der Gnosis: hier wie dort eine die Zeitgenossen in Staunen setzende geschichtliche Persöhnlichkeit, hier wie dort ihre göttliche Verehrung, hier wie dort ihre Umwertung 7u philosophischen Spekulationen. -

War aber Simon, die große Kraft Gottes, ein Zeitgenosse der Apostel, kein Wunder, dab die gnostische Lehre, die seinen Namen trug, weil sie ihn selber. zum Gegenstand hatte, einem Manne wie Justin der, mitten im Strom der christlich-gnostischen Bewegung stand, als der Anfang aller, auch der christlichen Gnosis und Simon selbst als der Archihäretiker erschien.

So ist es gekommen, daß zuerst Justin alle Häresien auf Simon zurückgeführt hat. $\mathrm{Da}$ nun auf dieser historisch unhaltbaren, aber zeitgeschicht- 
lich wohl verständlichen Geschichtsauffassung die nachfolgenden Häreseologen ihre Darstellungen aufbauten, erschien Simon auch geeignet, der Repräsentant der verschiedenartigsten antikirchlichen und -christlichen Richtungen in jener Zeit der werdenden katholischen Kirche zu sein. Doch ist es erst der am Anfang des 3. Jahrhunderts lebende Verfasser der Klementinen gewesen, der ihn literarisch dazu verwendete, nicht etwa weil er in Simon bezw. dem simonianischen System alle diese $Z$ uige wieder gefunden hätte, sondern weil er in einer Persönlichkeit alle diejenigen geistigen Richtungen seiner Zeit darstellen und niederlegen wollte, mit denen die damalige Kirche zu kämpfen hatte, vor allem die Gnosis und das Heidentum. Daß dabei der Simon der Klementinen neben den Zügen eines historischen Magiers auch die eines falschen Messias, neben denen des samaritanischen gnostischen Simonianismus auch die des alexandrinischen, neben denen des judenfeindlichen Paulus auch die des Marcion, neben denen eines astrologischen Fatalisten auch die eines philosophisch gebildeten Heiden trägt, das alles beruht nicht auf Absicht, sondern auf Zufall. Und wie die, willkürliche Verwertung heterogener Literaturwerke dieses phantastische 'Simonsbild gebildet, so ist die literarische Quellenkritik das Mittel, dieses vielköpfige Bild in seiner Zusammensetzung aufzuweisen und zu zeigen, was daran genuin simonianisch und was spätere Zutat ist. ${ }^{x}$

i In neuerer Zeit ist darauf hingewiesen worden, daß Simon sowohl in den Sibyllinen (vgl. Preuschen, Zeitschr. f. neutest. Wiss. 1901, $173 \mathrm{ff}$. W. Schmiedel, Art. Simon Magus in Encyclop. Brit. p. 4546 sq) als auch in der Apocal. Joh 13, 11-17 cf. 16, 13 19, 20 20, 10 (vgl. Spitta, Offenbarung des Joh. Halle $1889380 \mathrm{ff}$. Erbes, Offenbarung des Joh. Gotha 1891 25 ff.; Schmiedel 1. c. p. 4547) als Antichrist dargestellt werde. Indem wir auf diese Frage nicht näher eingehen, bemerken wir nur, daß eine derartige Identifizierung Simons mit dem Antichrist an und fur sich nichts Unwahrscheinliches an sich hat, da $\$$ aber dieser Antichrist Simon mit dem Antichrist Paulus nichts zu tun hat, den Preuschen in der Apocal. Elias (Zeitschr. f. neutest. Wiss. Ig01. 169 ff.) glaubt nachweisen zu können. 